Instituto Internacional de Investigación y Desarrollo Tecnológico Educativo INDTEC, C.A.

DOI: https://doi.org/10.29394/Scientific.issn.2542-2987.2020.5.16.8.162-178

OAI-PMH: http://www.indteca.com/ojs/index.php/Revista Scientific/oai

Artículo Original / Original Article

\title{
Aproximación Teórica de la Concepción del Gerente Agrícola: Una Visión Transcompleja (Zona del Páramo, Mérida)
}

\author{
Autora: María Teresa Rodríguez \\ Universidad Fermín Toro, UFT \\ mariateresarodriguez841@gmail.com \\ Mérida, Venezuela \\ https://orcid.org/0000-0003-1746-623X
}

\section{Resumen}

El presente artículo ha sido elaborado con la intención de realizar una investigación teórica del comportamiento del gerente agrícola en la zona del páramo de Mérida. El sector agrícola venezolano, ha cambiado en los últimos años, por lo tanto, necesita de un buen gerente para afrontar estos cambios. Como objetivo general, se pretende analizar una aproximación teórica del gerente agrícola desde un enfoque hermenéutico, una teoría transcompleja, y el objetivo específico es analizar los fundamentos teóricos filosóficos bajo un enfoque hermenéutico para mejorar la calidad del gerente agrícola aplicando un modelo epistémico subjetivo, y descubrir el estilo del pensamiento del gerente agrícola para mejorar sus funciones. La investigación es documental bajo un enfoque cualitativo, empleando para su desarrollo una metodología hermenéutica. La revisión y el análisis de los antecedentes históricos, investigativos, y teóricos. Por último, se analizan los resultados obtenidos y conclusiones determinando la calidad del gerente agrícola.

Palabras clave: agricultura; gerente; desarrollo agrícola.

Cómo citar este artículo:

Rodríguez, M. (2020). Aproximación Teórica de la Concepción del Gerente Agrícola: Una Visión Transcompleja (Zona del Páramo, Mérida). Revista Scientific, 5(16), 162-178, e-ISSN: 2542-2987. Recuperado de: $\underline{\text { https://doi.org/10.29394/Scientific.issn.2542-2987.2020.5.16.8.162-178 }}$

Fecha de Recepción: 12-07-2019
Fecha de Aceptación:

28-01-2020
Fecha de Publicación:

05-05-2020 
OAI-PMH: http://www.indteca.com/ojs/index.php/Revista_Scientific/oai

Artículo Original / Original Article

\title{
Theoretical Approach to the Conception of the Agricultural Manager: A Trans-Complex Vision (Wasteland area, Mérida)
}

\begin{abstract}
This article has been prepared with the intention of conducting a theoretical investigation of the behavior of the agricultural manager in the area of the Mérida wasteland. The Venezuelan agricultural sector has changed in recent years, therefore, it needs a good manager to face these changes. As a general objective, it is intended to analyze a theoretical approach of the agricultural manager from a hermeneutical approach, a trans-complex theory, and the specific objective is to analyze the theoretical philosophical foundations under a hermeneutical approach to improve the quality of the agricultural manager by applying a subjective epistemic model, and discover the style of thinking of the agricultural manager to improve his functions. The research is documentary under a qualitative approach, using a hermeneutical methodology for its development. Review and analysis of historical, research, and theoretical background. Finally, the results obtained and conclusions are analyzed determining the quality of the agricultural manager.
\end{abstract}

Keywords: agriculture; manager; agricultural development.

How to cite this article:

Rodríguez, M. (2020). Theoretical Approach to the Conception of the Agricultural Manager: A Trans-Complex Vision (Wasteland area, Mérida). Revista Scientific, 5(16), 162-178, e-ISSN: 25422987. Recovered from: https://doi.org/10.29394/Scientific.issn.2542-2987.2020.5.16.8.162-178

Date Received:

12-07-2019
Date Acceptance:

28-01-2020
Date Publication: 05-05-2020 


\section{Introducción}

Analizar la gerencia agrícola en la zona del páramo de Mérida. Se conduce bajo un enfoque cualitativo, empleando para su desarrollo una metodología hermenéutica. El sector agrícola en Venezuela, visto como una organización ha cambiado en los últimos años, tanto en lo político, económico como en lo social, para afrontar estos cambios necesita de un buen gerente que contribuya en su desarrollo; para ello, se analizará un modelo epistémico subjetivo.

Descubrir el estilo del pensamiento agrícola, para transformar el talento humano en sus funciones específicas y con el personal a su cargo. En los históricos se revisará la gerencia agrícola a principios del Siglo XX y XXI y las funciones del gerente en cada periodo en la zona del páramo de Mérida. En los investigativos, en el ámbito internacional, la tesis doctoral de Morantes (2014a): centrada en los sistemas de producción con ovinos en Castilla-La Mancha, aplicada en España; y en lo nacional se cuenta con el artículo de Timaure y Plata (2011a): en "[...] el desarrollo rural, así como los avances en materia de calidad de vida y productividad en la actividad agropecuaria [...]" (pág. 19). En los teóricos: Meditaciones Gerenciales de Barroso (1999a); y Gerencia Estratégica: Herramienta para la Toma de Decisiones en las Organizaciones de González, Salazar, Ortiz y Verdugo (2019a).

La metodología aplicada es el enfoque cualitativo en el que se desenvolverán la investigación, se basa en comprender el comportamiento, por lo que este momento del discurso, se considera oportuno puntualizar los referidos a Martínez (2011a): a que "el método básico de toda ciencia es la observación de los datos o hechos y a la interpretación (hermenéutica) de su significado" (pág. 18); esto implica que tanto la observación como la interpretación son inherentes, es decir se entrelazan, por lo tanto, las ciencias tratan de desarrollar observaciones sistemáticas y responder a la interpretación respectiva. Por último, se realizó una revisión literaria, 
información documental y autores claves.

El sector agrícola de los páramos andinos, se ha visto como una organización que ha venido cambiado en los últimos años, necesita ser gerenciado eficiente y eficazmente para poder manejar y solucionar los problemas que se estén presentando, como por ejemplo: económicos (costos de producción, tasas de interés); sociales y políticos (precios justos, subsidios, protección fiscal). Todo esto tiene mayor ventaja con un gerente agrícola eficiente.

Una de las referencias más antiguas acerca de la administración de fincas en el municipio Cardenal Quintero corresponde a la finca de la posesión en la cual se construyó la casa de la Cuchilla estancia principal de la hacienda perteneciente a la familia del señor Vitaliano Peña, la cual se ubicaba para finales de los años 30 en el actual sector de Aracay, y en la cual se sembraban hortalizas bajo un circuito económico cerrado. Es decir, todo lo que se necesitaba en la unidad de producción allí misma se elaboraba o se elaboraba o se intercambiaba en sus alrededores.

En diferentes espacios de esta finca de enormes proporciones se cosechaban papas, y trigo, en las veguillas se producian verduras, caraotas, arvejas, y otros frutos menores. En "El Rincón" sembraban apios, papas, maíz en tres variedades (cariaco despegado, cariaco chiquito del cual obtenían tres cosechas al año y maíz Yucatán. Además, se sembraban garbanzos, higos, ajo, hinojo, y plantaciones de "juquián" conocido comúnmente "sagú" (producto del cual se obtiene un almidón similar a la yuca) el cual crecía al lado de los apios, así como se producía el "churi" y el "zapallo" (son voces lugareñas de ascendencia indígena, es una planta rastrera que produce dos especies diferentes de calabazas, pertenecientes a una misma familia botánica).

Los trabajos de la finca eran cumplidos por peones, quienes se encargaban de las labores más forzadas, existían los cabresteros, su función 
es la de cuidar los animales y curarlos cuando era necesario. Los trabajadores tenían garantizada una buena alimentación, conformada por desayuno, almuerzo y cena, aunque se les servía en ambientes de la casa diferentes a los cuales utilizaba la familia para comer. Aun así, consumían productos sanos, pues para sembrar no se utilizaban agroquímicos.

Las mujeres se encargaban de las labores de ordeño y del hogar, que se hacía de forma colectiva pues, los productos lácteos obtenidos eran consumidos principalmente por la familia y por los empleados de la hacienda, por esta razón el trabajo realizado producía satisfacción, y sólo con unas pocas vacas se garantizaba el alimento de todos. Haciendo la salvedad de que estas mujeres no tenían acceso a la educación.

En relación a la obtención de las semillas debemos acotar que en los patios y corredores de la casa se asoleaban los productos agrícolas cosechados para luego sacar de allí las semillas que posteriormente iban a ser sembradas en las diferentes partes de la finca. Respecto a los aspectos históricos, Moreno (1996), señala que:

En épocas de la dictadura de Juan Vicente Gómez el señor Vitaliano Peña dio refugio a perseguidos y guerrilleros provenientes de Jajó, a familiares y gente leal al General Juan Araujo, quien había participado en un alzamiento militar contra el régimen (pág. 108).

Tras la muerte del dueño de la hacienda en la Cuchilla, los capataces se fueron del lugar, siguiendo las normas y costumbres de la época, que seguían como ley, pues, una mujer viuda no podía alojar a personas extrañas a su familia en la casa y debido a ello la finca entra en un proceso de declive, a esta situación se le suma la disgregación ocasionada en la familia por la participación de la herencia. Hasta la muerte de Vitaliano Peña (1865-1918), la finca perteneció a una misma familia. En la actualidad han ocurrido participaciones en diversos sectores de Aracay. 
Respecto a la tenencia de la tierra, los sectores Aracay y La Cuchilla eran desde tiempos de las encomiendas posesión de una sola familia. A finales del Siglo XIX los coherentes hacían repartición de tierras por documentos privados, autenticados por el juzgado del municipio y más tarde protocolizados en el registro subalterno del distrito.

En el caso de la hacienda de la Cuchilla y en los muchos otros, cada familia tiene su finca en la posesión, cuando no se realiza la partición de la herencia la posesión pertenece a varias familias, quienes de mutuo acuerdo establecen los limites convencionales sin firmar ningún documento, solo fijan las áreas comunitarias tales como, los porteros, y las áreas de siembra son de uso individual, cuando ya reparten y están de acuerdo entonces es el momento apropiado pata hacer el documento, para así legalizar el convenio.

A finales del Siglo $X X$, encontramos una segunda referencia respecto a la administración de una nueva finca en la parroquia Las Piedras, ubicada en el sector "El Estafiche", la cual perteneció al coronel del ejército Jesús Manuel Santiago, egresado de la Academia Militar de la Guardia Nacional Bolivariana (EFOFAC), quien en la década de los años 70, adquiere una finca que en el transcurso del tiempo llegó a ocupar una extensión de treinta hectáreas. Al inicio el dueño dirigía su hacienda y trabajaba con peones, sembrando hortalizas, papas, zanahorias, repollo, maíz, cilantro, y cebollín. Tiene sistema de rego privado, teléfono y otros medios de comunicación, y cuenta con todos los servicios públicos.

Durante los primeros años, sembraban con bueyes y poco a poco fue tecnificando su hacienda (rotulado de la tierra con Tractores) asumió un proyecto para modernizar la infraestructura, mediante la construcción de galpones que hoy día le sirven a la comunidad del "Estafiche" como silos de abono, de semillas y de los productos cosechados.

La finca presta servicios con sus diferentes maquinarias y tractores para preparar la tierra antes de sembrar en las fincas vecinas cobrando solidarios 
precios. Así mismo el coronel Santiago, se ocupaba del mantenimiento de la vía de penetración agrícola hacia el sector del "Estafiche", e impulsó proyectos para la instalación de un nuevo sistema de riego en la comunidad, y una nueva escuela, finalmente comenzó a trabajar con medianeros como una estrategia necesaria en la mejora de la producción.

La familia del coronel no estaba residencia en la hacienda, ni tampoco compartían con sus trabajadores en el contexto familiar, pues su formación militar no lo permitía, solo tenía un empleado de confianza que prestaba servicios de mantenimiento y vigilancia, a quien se le había construido una casa separada o independiente de la casa principal. Compraba los insumos agrícolas tanto en agropecuarias públicas como privadas. Posee un lavadero de zanahoria y una laguna artificial para abastecer de agua el sistema de riego. Contaba con un maestro de obras y un ayudante en el área de construcción para ampliar la casa principal.

La formación militar hizo el coronel Jesús Manuel Santiago, administrara su hacienda bajo un modelo económico abierto, moderno y tecnificado que le permitió aumentar el rendimiento de sus cultivos y por ende aumentar y mejorar su producción.

Entre las leyes en las cuales participó en su elaboración destacan: la Ley de Semillas y Material para la Reproducción Animal, Ley de Silos y Almacenamientos Agrícolas, Ley para Promover la Libre Competencia y La Eficacia; Ley Orgánica de Desarrollo Agrícola; Ley de Mercado Agrícola. Ley de Tierras y Desarrollo Agrícola, entre otras.

De acuerdo a lo antes expuesto, Paredes (2014), señala: "que las víctimas del deslave ocurrido en el sector de la primavera recibieron refugio en la finca del coronel Jesús Manuel Santiago, el lunes 03 de junio del año 2003 ocurrió un deslave proveniente de la quebrada la Sucia..." (pág. 257). Este deslave inundó las casas que fueron destruidas por la creciente, y 5 familias sobrevivientes a la tragedia se refugiaron en un galpón de la hacienda del 
coronel, ubicada en el sector "El Estafiche".

\subsection{Enfoque Investigativo}

Expone Morantes (2014b), en su tesis doctoral titulada Análisis de Gestión y Eficiencia en los Sistemas de Producción con Ovinos en Castilla-La Mancha, España, en la que:

Se estudió el desempeño gerencial y la implementación de estrategias de manejo en las ganaderías a partir de la elaboración de índices sintéticos, considerando las funciones de Planificación, Organización, Dirección y Control, y las prácticas y tecnologías factibles de realizar en el largo y corto plazo...Se evaluaron los resultados productivos de las ganaderías a través de las productividades parciales y se establecieron dos grupos de fincas, denominadas de alto y bajo nivel tecnológico (173).

Esta tesis corrobora la similitud con el artículo, porque refleja como mejora la gerencia en la zona del Páramo implementando las nuevas tecnologías y de esta manera mejora su productividad y eficiencia en la actividad agrícola.

Afirman Timaure y Plata (2011b), en su artículo sobre la Gerencia Participativa y Sostenibilidad en Comunidades Agrícolas, que:

Las características de sostenibilidad de las comunidades agrícolas estudiadas, conforman un conjunto de situaciones relacionadas, de acuerdo al análisis, en las Subdimensiones: social, económica, ambiental e institucional, expresando fundamentalmente, la potencialidad de estas comunidades para posicionarse en el tiempo, como líderes en producción agropecuaria a nivel local, porque tienen las condiciones adecuadas para su consolidación (pág. 30).

Este artículo se relaciona con la presente investigación porque los agricultores del páramo de Mérida tanto como los de la costa oriental del lago dependen varios factores entre ellos su entorno ambiental y social para su 
producción. De igual manera, los productores trabajan de manera individual y no en equipo que sería lo más idónea en pro de mejoras para su actividad.

\subsection{Supuestos Teóricos}

Según Barroso (1999b):

Los gerentes líderes llevan el timón. Los seguidores obedecen órdenes. Los líderes saben dónde ir y qué hacer. Los seguidores esperan órdenes permaneciendo inactivos. Esta diferencia es mucho más radical, no tan formal. Porque cada vez más las empresas necesitan gerentes que lideren los procesos, que diseñen e implanten la visión, que se comprometan con los valores y la cultura, y tomen decisiones, que den la cara (pág. 75).

Así como también los autores Kouzes y Posner (1993): escribieron un libro de contabilidad, intitulado "Credibilidad: cómo los líderes lo ganan y lo pierden" (pág. 76); en un mundo tan complejo en el que vivimos, el liderazgo ha pasado a ser compartido de vinculaciones y responsabilidades para mejorar los resultados, un estilo de liderazgo que posibilite y permita la participación, el compromiso y la identificación de todos los miembros de la empresa.

Las decisiones para que sean importantes deben tomarse en equipo, así los trabajadores asumen los éxitos y fracasos de la empresa y a tener responsabilidades en la complejidad de las decisiones gerenciales a enriquecer las responsabilidades en las oportunidades de crecimiento, compartir con los trabajadores y no hacerlo todo, dejar que ellos opinen y tomen algunas decisiones, les da oportunidades para enseñar lo que han aprendido por experiencia en la organización.

Existen varios modelos para desarrollar competencias, pero la más importante para este estudio es la gerencia estratégica por una visión compartida en las estrategias y la toma de decisiones. La gerencia del futuro es la que más asume riesgos se enfrenta a realidades para generar un mundo 
transcomplejo. Es por ello, la importancia del artículo sobre Gerencia Estratégica: Herramienta para la Toma de Decisiones en las Organizaciones, publicado por González, Salazar, Ortiz y Verdugo (2019b), señalan que el pensamiento estratégico:

Incorpora valores, misión y estrategia que tienden a ser elementos intuitivos, basados el sentimiento, más analítico, basados en información. Llegar a un acuerdo sobre estos elementos entre los miembros del equipo administrativo es un prerrequisito esencial para la planeación estratégica (pág. 244).

Siguiendo este orden de ideas, González, Salazar, Ortiz y Verdugo (2019c), plasman la importancia del pensamiento estratégico aplicado a la gerencia estratégica y el:

...Desarrollo de la empresa en su entorno o medio ambiente, conociendo, desarrollando y aplicando la gerencia estratégica como un arma poderosa en el mundo competitivo de hoy [...] el gerente debe asumirse como un agente de cambio y de transformación, que conduzca a las organizaciones por nuevos rumbos, nuevos procesos, nuevos objetivos, nuevas estrategias, nuevas tecnologías e incluso nuevos riesgos; un agente que con su dirección y orientación, modifique comportamientos y actitudes del capital humano, un agente cultural que, con su estilo de gerencia, reforme y difunda la cultura organizacional, utilizando técnicas de desarrollo gerencial dirigidas a incrementar la habilidad del liderazgo y resaltar los valores institucionales (pág. 245).

En este artículo, se hace acotación a través de una gerencia estratégica, en la que cualquier empresa podrá alcanzar el éxito si toma en cuenta todos los factores en la toma de decisiones, es por ello, que para mejorar la empresa agrícola se debe tomar en consideración que, en el proceso de planeación estratégica, todos los puntos de vista son importantes como en lo económico, social, técnico, político, cultural, y el entorno. Por ende, mejora la productividad del sector agropecuario a mediano y largo plazo. Como objetivo, se planteó realizar una aproximación del gerente agrícola en la zona 
del páramo de Mérida, bajo una visión transcompleja y analizar los fundamentos teóricos, históricos y filosóficos, desde un enfoque hermenéutico, para mejorar la calidad del gerente agrícola, bajo un modelo epistémico subjetivo.

\section{Metodología}

El enfoque cualitativo en el que se desenvolverá la investigación, se basa en comprender el comportamiento, por lo que este momento del discurso, se considera oportuno puntualizar, que para Martínez (2011b):

El método básico de toda ciencia es la observación de los datos o hechos y a la interpretación son inherentes, es decir, se entrelazan. Por lo tanto, las ciencias tratan de desarrollar observaciones sistemáticas y responder a la interpretación respectiva (pág. 66).

Por otra parte, con el fin de recolectar la información, se hace lo necesario la utilización de un diseño de investigación que para esta ocasión es de campo, el cual según Sabino (1992): "se basan en informaciones o datos primarios, obtenidos directamente de la realidad" (pág. 100); para el estudio el diseño de campo por los agricultores de la zona del páramo de Mérida, considerado la extensión de zona en producción y los rubros agrícolas.

Por último, la investigación estará también contemplada por las siguientes fases:

- Revisión literaria relacionada con el tema de estudio (exploración).

- Ordenamiento de la información documental recabada (evaluativa).

- Actores Sociales (informantes claves) los agricultores de la zona en estudio.

- Técnicas e instrumentos: basados en la técnica de la entrevista aplicada a los informantes claves de la investigación.

- Categorización de la información: la misma se desarrolla para dar paso 
a una aproximación teórica.

\section{Resultados}

A través de las encuestas realizadas, se pudo constatar, lo que el Instituto de Desarrollo Agropecuario (INDAP, 1997), plantea en su libro de Gestión para la modernización de la pequeña empresa agrícola, que: "la agricultura se ha visto desfavorecida por el escenario de mayor inestabilidad cambiaria" (pág. 78); afectada no solo por lo económico, sino también por lo político y social, pues las empresas agrícolas enfrentar el reto de modernización de su gerencia para estar al nivel en este medio tan competitivo y que amerita "la actualización del recurso humano, como factor de primera importancia en cuanto a sustentar la capacidad competitiva de la empresa" (pág. 77).

Por lo tanto, es de suma importancia que los productores agrícolas de la zona del páramo para tener una alta productividad a nivel gerencial debe ser complementada con una gerencia de calidad, se concluye preliminarmente que la meta de la misma en el páramo andino es un reto, ya que, el conocimiento en la mayoría de los productores de cómo dirigir y ser competitivo en el mercado nacional es experimental, pues los mismo, van aprendiendo de su propio negocio, a través del tiempo del ensayo y error.

Por ende, la decidida voluntad de nuestros productores transformados en gerentes agrícola permitirá, enfrentar con éxito la apertura comercial para garantizar la suficiencia alimentaria que el país requiere. De esta manera, Pérez (2019), en su artículo publicado en la Revista Scientific, con el título de Hermenéutica de la Flexibilidad Cognitiva de las Decisiones Estratégicas Empresariales desde la Transcomplejidad, indica que:

El proceso de toma de decisiones estratégicas como fenómeno exhibe características complejas, puesto que conecta relaciones, intereses, contradicciones, riesgos y beneficios. Superada la tesis del actuar racional como patrón universal del 
comportamiento de los agentes de la economía social, entre los cuales los gerentes juegan un papel esencial, las decisiones se encuentran insertas en la dinámica social organizacional, cuyo conocimiento parece necesario a la hora de comprender los cimientos y pilares que guían el proceso de decisiones estratégicas de los directores ejecutivos (pág. 140).

Es por ello, que a través de la cita anterior, las decisiones gerenciales son importantes para la toma decisiones, pues no solo se rige por los conocimientos, estrategias y políticas sino también debe tomar en cuenta su entorno y la interacción social donde se encuentra su empresa. En el caso estudio, los productores además del conocimiento experimental deben aplicar también conocimientos gerenciales y de toma de decisiones que contribuya al mejoramiento y productividad agrícola.

En entrevista realizada a ocho (08) productores (informantes clave) de la zona del páramo de Mérida, se obtuvieron los resultados que se muestran en la tabla 1, en este caso se les consultó a los productores cuáles eran los principales rubros agrícolas en producción.

Tabla 1. Los principales rubros agrícolas de producción son:

\begin{tabular}{|l|l|l|l|l|}
\hline Rubros & \multicolumn{1}{|c|}{ Tiempo } & $\begin{array}{c}\text { Condiciones } \\
\text { Climáticas }\end{array}$ & \multicolumn{1}{c|}{ Altitud } & \multicolumn{1}{c|}{ Producción } \\
\hline Papa & 3 meses & Verano-riego & 1700 a $2000 \mathrm{mts}$ & $\begin{array}{l}\text { 1ha de semilla de } 10 \text { a } 15 \\
\text { veces el producto. }\end{array}$ \\
\hline Zanahoria & 3 meses & Verano-riego & 1700 a $1900 \mathrm{mts}$ & $\begin{array}{l}100 \text { grs de semilla da } \\
\text { aproximadamente } 10 \mathrm{~kg} \\
\text { del producto. }\end{array}$ \\
\hline Cilantro & 1 a 2 meses & Invierno & & $\begin{array}{l}1 \text { grs de semilla da } 1 \mathrm{~kg} \text { del } \\
\text { producto. }\end{array}$ \\
\hline Lechuga & 2 meses & Invierno & & \\
\hline Pimentón & 1 a 2 meses & Verano & & \\
\hline
\end{tabular}

Fuente: La Autora (2019).

Los principales rubros agrícolas que se producen en la zona del páramo merideño son: la papa, la zanahoria, que se cultivan en periodos largos que 
van desde octubre a enero (época de verano y el mantenimiento del cultivo es con riego) y los rubros como: cilantro, lechuga, pimentón, entre otros, son de periodos cortos y se cultivan en cualquier época del año.

\subsection{Problemas más comunes en la producción:}

- Los sistemas de riego son comunes para toda la zona, se han realizado proyectos de sistemas de riego, pero no han sido realizados.

- La materia prima, los productores tienen que adquirir los insumos primero para sembrar, de lo contrario no pueden producir, no pueden correr el riego de no encontrarlos a tiempo y pierdan la cosecha.

- La mano de obrar calificada es muy escasa, laboran los colombianos y personal de otras zonas.

- La maquinaria agrícola es limitada solo unas pocas empresas agropecuarias tienen y las alquilan a en un tiempo muy limitado.

- Las cosechas se realizan dos veces al año, los pequeños agricultores a veces realizan una sola cosecha por la dificultad para adquirir los insumos.

- Los sistemas de ventas de la producción.

- La mayoría de las veces venden en las fincas. Y cancelan sus productos en un máximo de 5 días.

- Los que producen en gran escala venden sus productos a regiones vecinas (Barinas y Mérida) y otros clientes los llevan al centro.

\section{Reflexiones Finales}

En el Siglo XIX las fincas eran unidades de producción locales, lo cual incidía en la producción de rubros que permitían la subsistencia de la comunidad. Los productos cosechados como el maíz, duraban más de seis meses y no se dañaban. El salario de los trabajadores o peones en moneda 
era mínimo, se les daba alimentación y artículos de vestir para así cubrir sus necesidades básicas, se mantenían las diferencias marcadas en los diferentes estratos sociales, había poca movilidad social y por ende, no tenían acceso a la educación.

En la actualidad se les asigna a los trabajadores un pago, por el día de trabajo, pago por tarea, o por contrato, siendo esta última modalidad la que se da con menor frecuencia. Se les da alimentación: desayuno y almuerzo, y ahora los trabajadores tienen más acceso a la educación; sin embargo, de acuerdo al análisis arrojado en el estudio de estos dos casos nos sugieren que las diferencias de clases sociales aún son muy marcadas, en este sentido en ninguno de los dos casos señalados los dueños de la hacienda comparten con sus trabajadores.

Es necesario recomendarle al gerente agrícola, la importancia que tiene cumplir con las leyes del Seguro Social Obligatorio (IVSS) y el Fondo de Ahorro Obligatorio para la Vivienda (FAOV), para los trabajadores y trabajadoras de sus fincas.

\section{Referencias}

Barroso, M. (1999a,b). Meditaciones Gerenciales. ISBN 10: 980-6194-28-4; ISBN 13: 978-980-6194-28-1. Carcas, Venezuela: Editorial Galac. González, J., Salazar, F., Ortiz, R., \& Verdugo, D. (2019a,b,c). Gerencia estratégica: herramienta para la toma de decisiones en las organizaciones. Telos, 21(1), 242-267, ISSN: 1317-0570; e-ISSN: 2343-5763. Recuperado de:

http://ojs.urbe.edu/index.php/telos/article/view/3002 INDAP (1997). Gestión para la modernización de la pequeña empresa agrícola. ISBN 10: 956-7718-00-8; ISBN 13: 978-956-7718-00-9. Santiago, Chile: Instituto de Desarrollo Agropecuario, Ministerio de Agricultura. 
Kouzes, J., \& Posner, B. (1993). Credibility: How Leaders Gain and Lose It, Why People Demand It. San Francisco, United States: Jossey-Bass. Martínez, M. (2011a,b). Ciencia y arte en la metodología cualitativa. Caracas, Venezuela: Editorial Trillas.

Morantes, M. (2014a,b). Análisis de la gestión y eficiencia en los sistemas de producción con ovinos en Castilla-La Mancha, España. Tesis Doctoral. España: Servicio de Publicaciones de la Universidad de Córdoba, Recuperado de:

http://www.uco.es/zootecniaygestion/img/pictorex/18 $17 \quad 50$ Tesis Ma rtina Morantes.pdf

Moreno, E. (1996). Valparaíso de Las Piedras Cuatricentenaria. ISBN: 9802219983. Mérida, Venezuela: Ediciones del Rectorado de la Universidad de Los Andes.

Paredes, P. (2014). Las Piedras a través del tiempo. Segunda edición, ISBN: 978-980-12-3672-6. Mérida, Venezuela: Producciones Editoriales, C.A.

Pérez, M. (2019). Hermenéutica de la Flexibilidad Cognitiva de las Decisiones Estratégicas Empresariales desde la Transcomplejidad. Revista Scientific, 4(11), 138-155, e-ISSN: 25422987. Recuperado de: https://doi.org/10.29394/Scientific.issn.25422987.2019.4.11.7.138-155

Sabino, C. (1992). EI Proceso de Investigación. Edición totalmente corregida y actualizada. Caracas, Venezuela: Editorial Panapo.

Timaure, C., \& Plata, D. (2011a,b). Gerencia participativa y sostenibilidad en comunidades agrícolas. CICAG: Revista Electrónica Arbitrada del Centro de Investigación de Ciencias Administrativas y Gerenciales, 8(1), 19-32, e-ISSN: 1856-6189. Recuperado de:

http://ojs.urbe.edu/index.php/cicag/article/view/538 


\section{María Teresa Rodríguez}

e-mail: mariateresarodriguez841@gmail.com

Nacida en La Puerta, estado Trujillo, Venezuela, el 15 de octubre del año 1948. Desde el año 1980 hasta el año 1984, me desempeñé en el cargo de Analista de Proyectos Industriales II, en el Instituto Autónomo CORPOINDUSTRIA, Valera, estado Trujillo; En el periodo 1986 hasta 1989, ocupé el cargo de Coordinador de Ventas en Confecciones DANA, C.A., Valera, estado Trujillo; En los años 1990 al 1995, ejercí el cargo de Auditor de Contabilidad en el Departamento de Tesorería General de la Gobernación del estado Mérida; Y desde el año 1990 hasta a la fecha, ejerzo como jefe del departamento de Contabilidad en el Instituto Universitario de Tecnología "Antonio José de Sucre" (IUTAJS), extensión Mérida, Venezuela.

El contenido de este manuscrito se difunde bajo una Licencia de Creative Commons ReconocimientoNoComercial-Compartirlgual 4.0 Internacional 